\title{
In situ ESEM imaging of the vapor-pressure-dependent sublimation-induced morphology of ice
}

\author{
Malavika Nair, Anke Husmann, Ruth E. Cameron, ${ }^{*}$ and Serena M. Best ${ }^{\dagger}$ \\ Cambridge Centre for Medical Materials, Department of Materials Science and Metallurgy, University of Cambridge, \\ 27 Charles Babbage Road, Cambridge, CB3 OFS, United Kingdom
}

(Received 17 January 2018; revised manuscript received 15 March 2018; published 27 April 2018)

\begin{abstract}
Sublimation is a fundamental phase transition that has a profound impact on both natural phenomena and advanced manufacturing technologies. Although great strides have been made in the study of ice growth from melt and vapor, little consideration has been given to the effect of sublimation on the morphological features that develop in the ice microstructure. In this experimental study, we demonstrate the effect of vapor pressure on the mesoscopic faceting observed and show that a vapor-pressure-specific wavelength characterizes the periodic features that arise during sublimation. The ability to control the length scale of these features not only provides us with new insights into the mesoscopic roughness of ice crystals, but also presents the potential to exploit this effect in a plethora of applications from comet dating to ice-templated tissue engineering scaffolds.
\end{abstract}

DOI: 10.1103/PhysRevMaterials.2.040401

Introduction. Exploring the physics underpinning sublimation has become vital as the evidence for its impact continues to grow-from the flow of glacial Antarctic ice [1] and optical properties of cirrus clouds [2] to the purification of semiconductor materials [3] and freeze-casting of polymers and ceramics [4]. Sublimation can apply in virtually any solid system, but is most commonly observed in the natural world through ice. At ambient pressures and temperatures, ice exists in a region of the phase diagram where phase transformations between the solid, liquid, and vapor can readily occur, producing vastly different structures depending on the conditions of growth and desorption. Although great strides have been made in the study of ice growth from melt and vapor, little consideration has been given to the effect of sublimation on the morphological features that develop in the ice microstructure.

Previous work attempting to elucidate the mechanism for the sublimation of ice has routinely considered a single crystal under precise undersaturations. The macroscopic morphological development has been extremely well characterized at the millimeter to micrometer scale using optical microscopy, but has resulted in conflicting evidence with some studies reporting an overall rounding of crystals [5] while others demonstrate overall faceting [6]. The most common method of studying the crystal faces of ice involves generating an impression (the "formvar" technique) used to produce negative crystals of the ice. This technique was first conceived when crystallographic etch pits from sublimation of ice crystals were observed to reflect the intrinsic crystal structure [7] and has since been

\footnotetext{
*rec11@cam.ac.uk

${ }^{\dagger}$ smb51@cam.ac.uk
}

Published by the American Physical Society under the terms of the Creative Commons Attribution 4.0 International license. Further distribution of this work must maintain attribution to the author(s) and the published article's title, journal citation, and DOI. used to provide a wealth of information on the growth of single crystals and the relative stability of the various crystal faces of ice [8]. However, there is a need to move from the static, single crystalline and macroscopic imaging of ice to resolve features at a finer spatial and temporal resolution, in order to understand the mechanisms leading to crystallographic restructuring. This paper exploits this etch pit classification to dynamically image the ice under the environmental scanning electron microscope (ESEM) and study the evolving micro- and mesostructure during the sublimation of polycrystalline ice.

ESEM. Although conventional scanning electron microscopy often requires a high vacuum to obtain highresolution secondary electron images, the advent of ESEM has enabled good quality imaging of hydrated samples [9]. The ESEM operates by purging the chamber and flooding it with water vapor such that the chamber pressure is equivalent to the vapor pressure of water. ESEM has unique detectors that can then exploit the molecules of water vapor to act as a cascade amplifier for the secondary electrons ejected from the sample [10]. The use of ESEM therefore has allowed us to image the sublimation process at the interface of the mesoscopic and microscopic scales of various materials. This includes frost, or ice grown from vapor, where previous work has observed mesoscopic roughness [11] and nonequilibrium phenomena involved in the sublimation of frost, but no systematic measurements have been made on the dependence of such roughened features on external parameters. In spite of all the successes of the environmental scanning electron microscope, the microstructure of ice as it grows from melt has never been imaged under the ESEM. This is partially due to the inability to control the nonequilibrium transitions that manifest in the ESEM chamber around the triple point, which either results in repeated condensation of the warmer water condensing on frozen ice, or the direct formation of frost [see Supplemental Material (SM), Fig. 1 [12]] onto the stub from the vapor. In this work, we are able to exploit the purge and flood cycles of the ESEM to ensure that the vapor pressures and chamber pressures can be used interchangeably. 


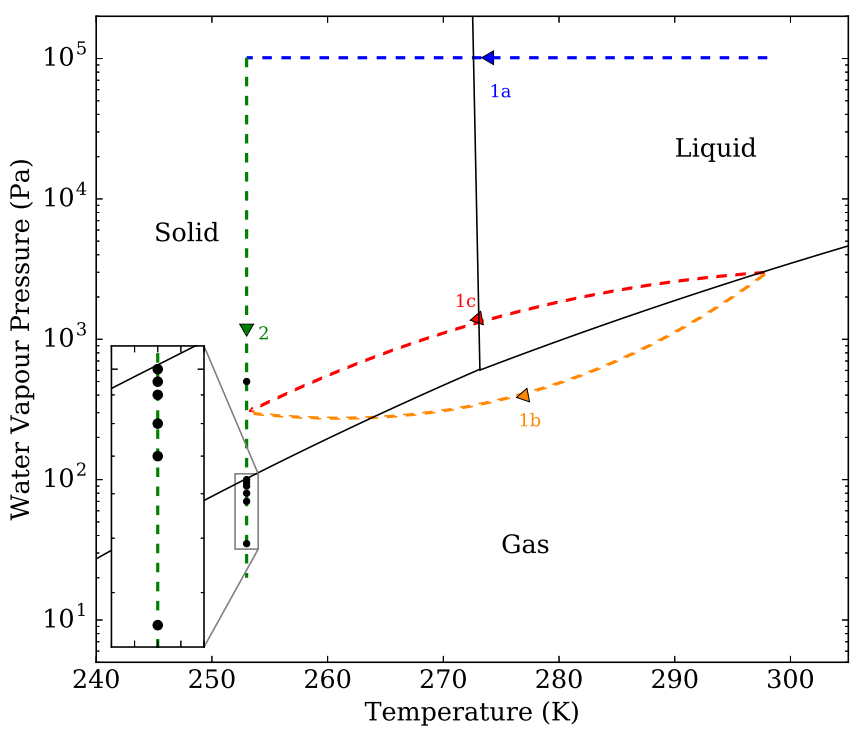

FIG. 1. Schematic of the freezing cycle employed in the ESEM chamber (blue and green), as well as deposition (red) and condensation (orange) nonequilibrium transitions that manifest in the ESEM chamber around the triple point. Stage 1a in the blue cycle allows the thermally driven growth of ice from melt thus ensuring that no intermediary transitions of water occur. The equivalent stage $1 \mathrm{~b}$ in the orange transition results in a solute-driven growth of frost from vapor, or condensation of warmer water atop the solidified ice in $1 \mathrm{c}$ of the red cycle. Stage 2 results in the solute-driven sublimation of any ice or frost. The black dots represent the seven pressures $(35,70$, $80,90,95,100,500 \mathrm{~Pa}$ ) considered in this study at $-20^{\circ} \mathrm{C}$.

Crystal growth and dynamics. Quantitative descriptions of the features that arise from some of such nonlinear phase transitions are well established. Fine atomic to nanoscopic steps and their interactions are routinely observed in deposition systems, for instance, in the formation of vicinal steps on $\mathrm{Si}$ [13] or GaAs [14]. These systems have a tendency to undergo step-bunching, often — but not necessarily_driven by impurities in an external flux [15], which lead to a range of submicron features observed during crystal growth. While many computational models now exist in this field, little experimental work has discovered morphology development to the same extent during evaporation.

On the microscopic to macrosopic scale, however, crystal growth can also be addressed through the consideration of instabilities on a front. The Mullins-Sekerka linear instability analysis, for instance, continues to be widely used in solidification processing of alloys where solute and thermal gradients lead to the development of dendritic sidebranching [16]. Specifically in the case of ice, phenomenological relationships have also been derived, linking the solidification front velocity to the size of the observed features [24,25]. Although much more challenging to reproduce in laboratory settings, the development of highly faceted snowflake arms have not only been reproduced exactly [17] but also the general problem (called the Stefan task) has been extensively modeled computationally [18-20]. Regardless, a quantitative description of morphology development in the solid to vapor system has not yet been determined at this scale.
Consequently, the work done in this paper contributes independently to the fields of sublimation dynamics and ice crystal growth by identifying high-resolution features that can be uniquely attributed to the sublimation of ice. Using a careful stepwise control of temperature and pressure to circumvent any nonequilibrium pathways as illustrated in Fig. 1, we can successfully image the structure of polycrystalline bulk ice during the sublimation process. We demonstrate that the pressure of sublimation chosen not only can introduce periodic features reflecting the intrinsic crystallography of ice, but also affects the time and length scale at which these features arise. These insights are then used to suggest a crystallographic mechanism exploring the formation of such sublimation-driven features. Since the vapor pressures can be considered independently, the results of this work can therefore be employed to consider the effects on ice under natural environments where precise undersaturations may not be known.

Methods. Aluminum ESEM stubs were filled with distilled water to a filling height of $1 \mathrm{~mm}$ and frozen using a Peltier stage to $-20^{\circ} \mathrm{C}$ at a rate of $1{ }^{\circ} \mathrm{C} \mathrm{min}{ }^{-1}$ at ambient pressure. Upon stabilization of temperature, the pressure was then reduced to various set values $(35,70,80,90,95,100$, and $500 \mathrm{~Pa})$, ensuring that minimum purge tolerance was above $700 \mathrm{~Pa}$ to ensure that the frozen sample neither melts nor sublimes during the pumping process. A thin layer of large single crystalline and highly faceted frost was formed on the surface of the solidified ice while the temperature equilibrated at atmospheric pressures. This layer of frost was allowed to fully sublime before capturing the time series representing the sublimation of the underlying ice. The images were captured with a GSED detector at a high voltage of $10 \mathrm{kV}$, beam current of $0.88 \mathrm{nA}$, and a frame rate of $30 \mu \mathrm{m} \mathrm{s}^{-1}$ at 500 and $100 \mathrm{~Pa}$, and $3 \mu \mathrm{m} \mathrm{s}^{-1}$ for all other conditions.

The mean sublimation velocities were calculated using the ImageJ MTrackJ plug-in to track the advancing sublimation front in each consecutive image. Using the ImageJ line measurement tool, the sublimation ridge spacings were determined by averaging features containing no fewer than four periods each across at least 20 ice crystals for each pressure condition studied. Measurement of ridge spacings have not been corrected for polycrystalline geometry effects, resulting in the negative skew of all distributions as seen in SM Fig. 3 [12]. Holding the same direction of skew has allowed the measured spacings to ensure consistency between measurements for the purpose of illustrating the effect of pressure on ridge spacings, although absolute accuracy is not achieved.

Results. The sublimation at $100 \mathrm{~Pa}$ of ice grown from melt at $-20^{\circ} \mathrm{C}$ was imaged over time to produce the micrograph series illustrated in Fig. 2 where several key morphological features can be noted. The sublimation process at $t_{0}$ initially results in a roughened surface with many "instabilities" dotted throughout the microstructure (see SM Fig. 2 [12] for labeled features). These instabilities begin to coalesce from $t_{1}$ through to $t_{4}$ where they form noticeable ridges of a characteristic and regular periodicity.

Crystallographic etch pits also arise at $t_{1}$ and $t_{2}$ at the initial stage of sublimation. These etch pits reveal a preference for the secondary prismatic face, as seen from the dotted white region in $t_{2}$, which is further expanded in Fig. 3. The classification of such etch pits to their corresponding crystal faces has been well studied, with the schematic in Fig. 3 relating the features 


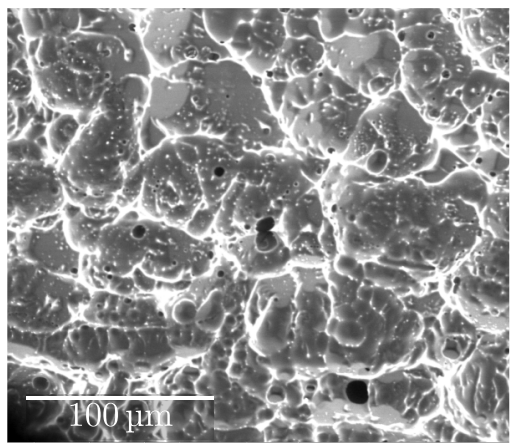

(a) $t_{0} ; 0 \mathrm{~min} 00 \mathrm{sec}$

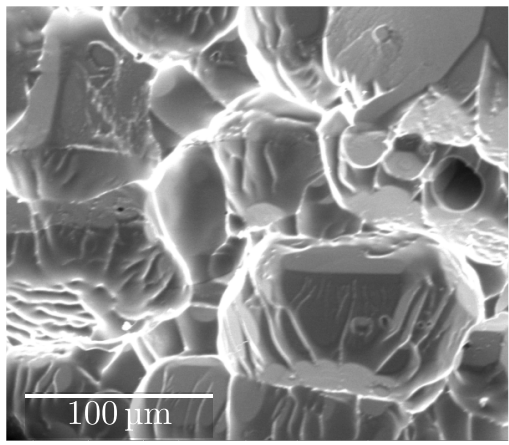

(d) $t_{3} ; 9 \min 43 \mathrm{sec}$

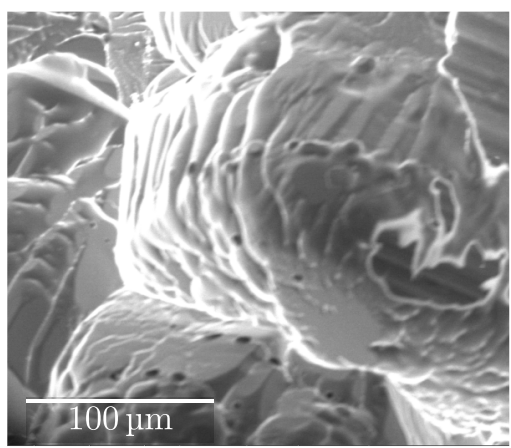

(g) $t_{6} ; 22 \min 3 \mathrm{sec}$

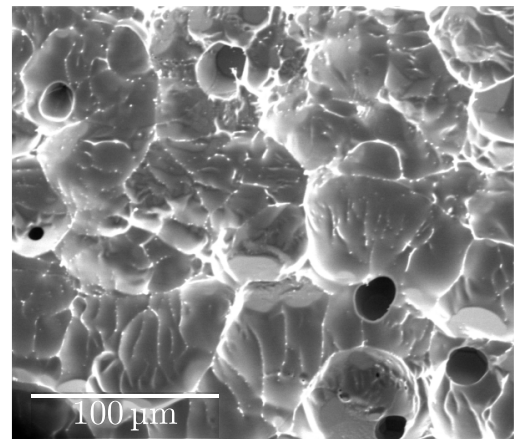

(b) $t_{1} ; 2 \mathrm{~min} 10 \mathrm{sec}$

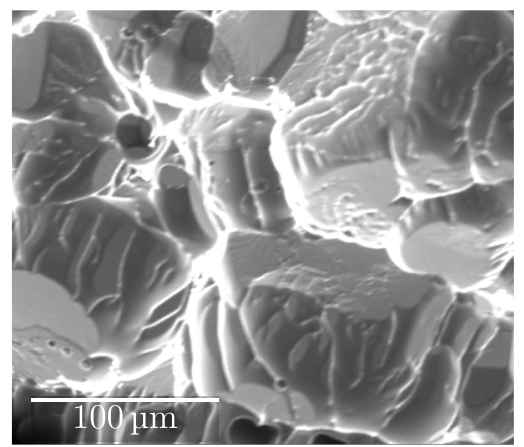

(e) $t_{4} ; 13 \mathrm{~min} 4 \mathrm{sec}$

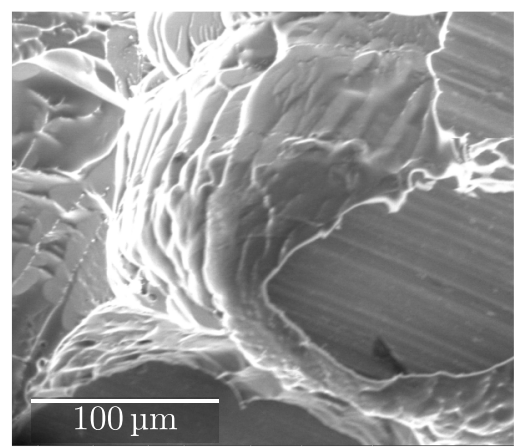

(h) $t_{7} ; 23 \mathrm{~min} 16 \mathrm{sec}$

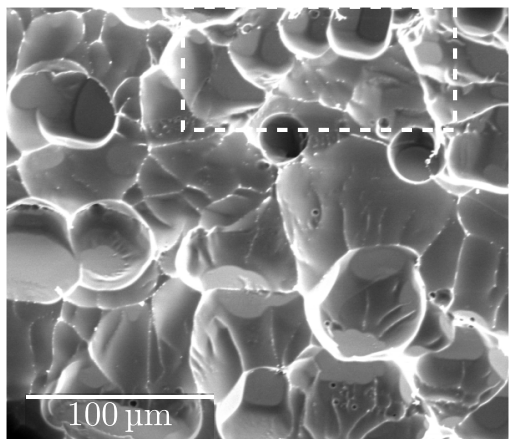

(c) $t_{2} ; 4 \min 19 \mathrm{sec}$

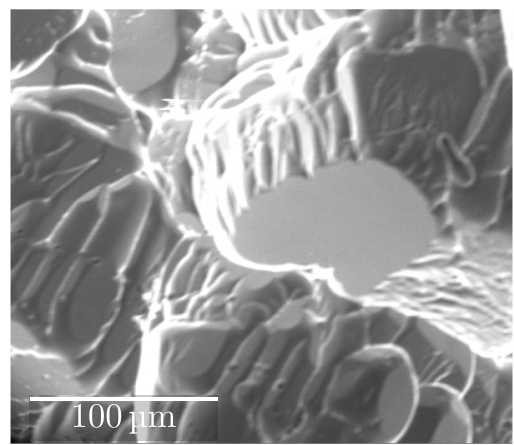

(f) $t_{5} ; 17 \mathrm{~min} 32 \mathrm{sec}$

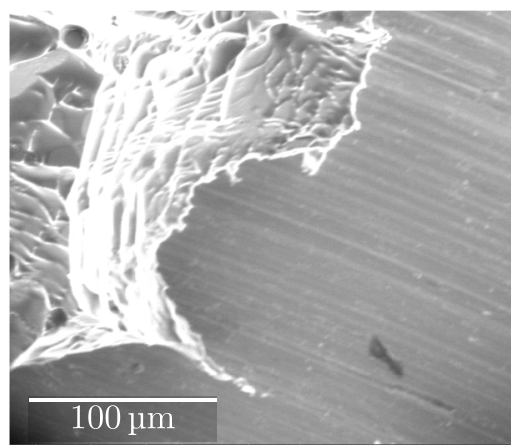

(i) $t_{8} ; 28 \min 6 \mathrm{sec}$

FIG. 2. Representative images from the time series of in situ sublimation of ice at $100 \mathrm{~Pa}$ at $-20{ }^{\circ} \mathrm{C}$ from initial time $t_{0}$ to final time $t_{8}$. Images are taken at time points where different stages of morphological development were distinctly noted, and hence do not represent evenly spaced time points.

observed in our ESEM microstructures to the various crystal planes in ice.

Even though the growth conditions for these ice crystals are not strictly comparable, this result is consistent with the findings by Shultz et al. [8], which suggest that the secondary prismatic plane is the preferred face for ice growth from melt, followed by the primary prismatic plane with the basal plane trailing far behind. From $t_{3}$, the microstructure of etch pits have sublimed deeper revealing a characteristic hexagonal shape. This may be attributed to the fact that the basal plane is the most stable crystallographic face in the context of the ice-vapor system [8].
Tracking the development of the secondary prismatic etch pits from $t_{2}$ to $t_{5}$, these pits proceed to expose the smooth basal planes with periodic ridges that consistently form perpendicular to the basal plane and travel approximately $30^{\circ}$ away from the $a$ or $b$ axis, i.e., along the secondary prismatic planes. The final stages of sublimation result in the eventual removal of the smooth basal planes as seen from $t_{6}$ to $t_{8}$. As the bulk of the crystal hollows out, the slower subliming secondary prismatic planes persist, with the final sublimation front moving perpendicular to the prismatic ridges.

At $-20^{\circ} \mathrm{C}$, the equilibrium water vapor pressure for the ice-vapor transition was determined to be 103.2 Pa using the 


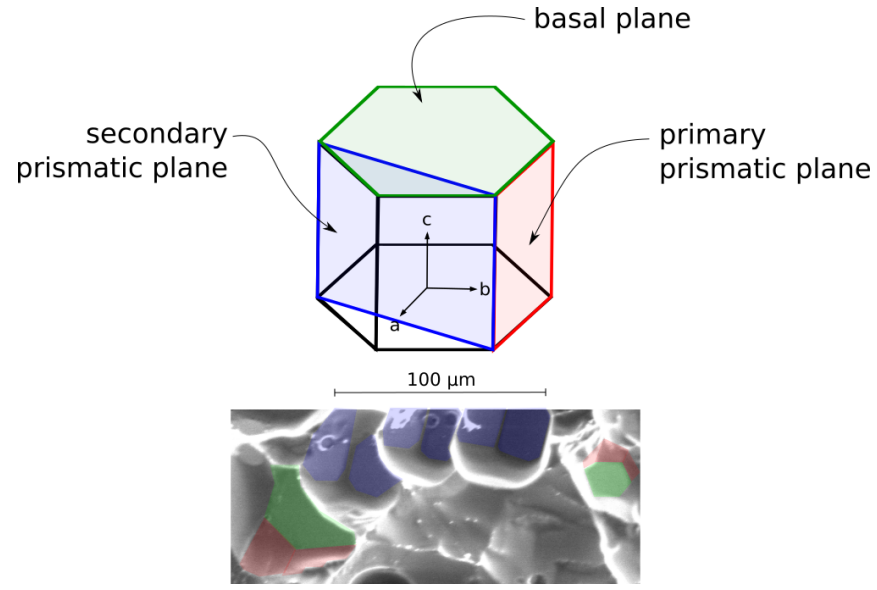

FIG. 3. Common crystallographic etch pits as observed in the ESEM and their corresponding (0001) basal (green), $\{10 \overline{1} 0\}$ type primary prismatic (red), and $\{11 \overline{2} 0\}$ type secondary prismatic (blue) crystal faces. ESEM snapshot is from the white dotted region in $t_{2}$ of the $100 \mathrm{~Pa}$ time series.

empirical relationship by Wexler [21]. Subliming ice grown under identical conditions at different vapor pressures, however, reveals further features of interest as seen in Fig. 4, where two conditions are below the equilibrium pressure and one above.

The general sublimation behaviors at the two vapor pressures below equilibrium are nearly identical, with prismatic etch pits arising at the early stages, followed by ridge formation and the hollowing of basal planes. At pressures higher than this equilibrium, it can be seen that the crystal surface front moves smoothly, driven primarily by the desorption of surface atoms, thus revealing etch pits and nonuniform local sublimation along the grain boundaries of the crystal. The etch pits are significantly more faceted at lower pressures than the smooth fronts observed at higher pressures. Furthermore, the periodic ridges observed at $35 \mathrm{~Pa}$ are finer in scale than at $100 \mathrm{~Pa}$, and completely unobserved at $500 \mathrm{~Pa}$. The habit of the ridges observed in this work is also consistent with evaporation steps as observed by Furukawa [22], where a forced pressure differential is applied to the center of a single crystal as it is hollowed out from the inside. The evaporation steps, however, are millimeters apart and occur over very large time scales (20 s) unlike the mesoscopic roughness observed here but akin to the surface restructuring noted at $500 \mathrm{~Pa}$.

To quantify the observations, a phenomenological relationship between the deviation from the equilibrium vapor pressure and the wavelengths observed was obtained through a systematic study at six pressure conditions under the equilibrium vapor pressure as illustrated in Fig. 5.

Linear regression on the data suggests a model where $\lambda_{o}=25 \Delta P^{-0.4}$, where $\lambda_{o}$ is in $\mu \mathrm{m}$ and $\Delta P$ in $\mathrm{Pa}$, indicating that larger deviations from equilibrium pressures are highly correlated with a refinement of the observed ridge wavelengths, with a suggested power-law relationship between the pressure deviation $\Delta P$ and ridge wavelengths $\lambda_{o}$.

Such power-law relationships are not uncommon in characterizing the periodicity of instabilities that develop in phase transitions. In liquid-solid systems, the solidification front velocity $V$ has been used to predict the grain size using various

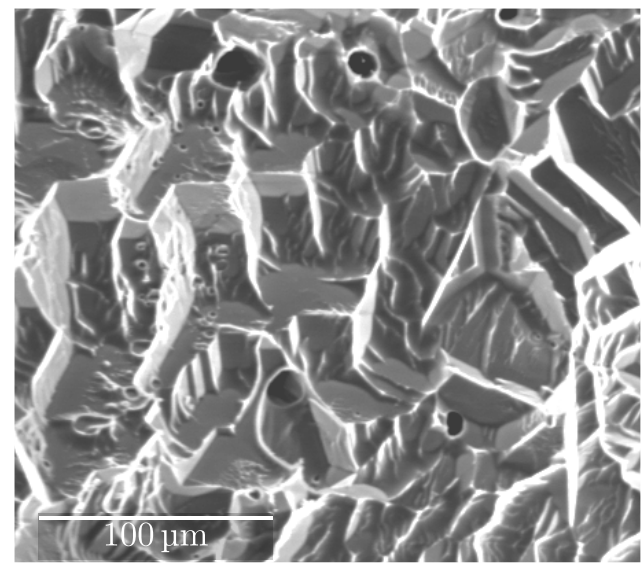

(a) $\mathrm{P}=35 \mathrm{~Pa}, \Delta \mathrm{P}=68.2 \mathrm{~Pa}$

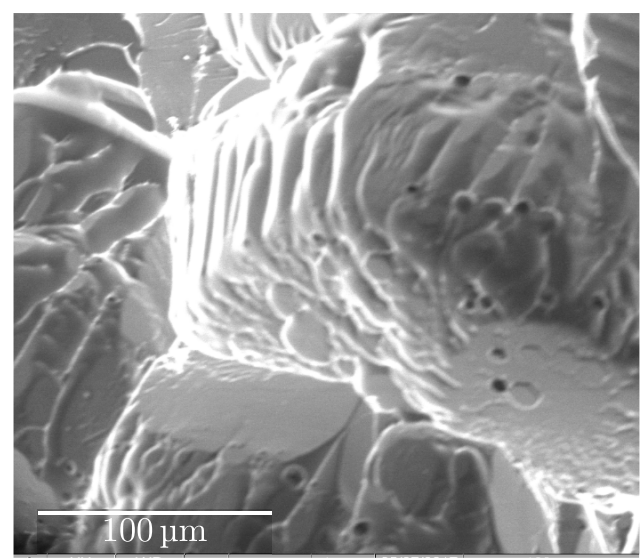

(b) $\mathrm{P}=100 \mathrm{~Pa}, \Delta \mathrm{P}=3.2 \mathrm{~Pa}$

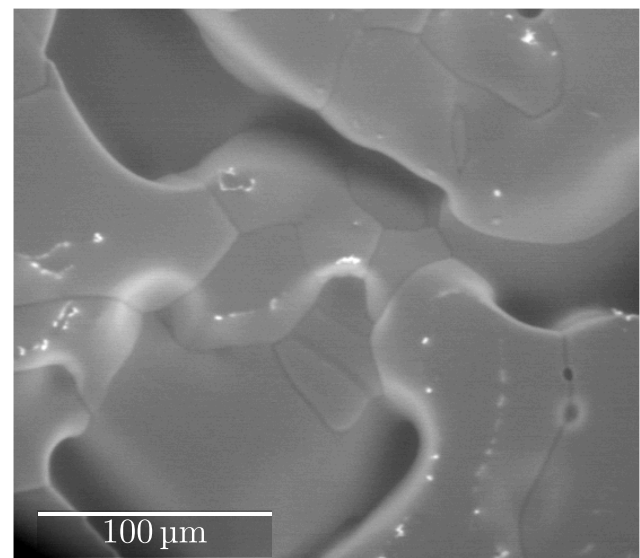

(c) $\mathrm{P}=500 \mathrm{~Pa}, \Delta \mathrm{P}=-396.8 \mathrm{~Pa}$

FIG. 4. Comparison of the ridge spacings in ice at $-20^{\circ} \mathrm{C}$ sublimed at chamber (or water vapor) pressures $P$ of 35,100 , and $500 \mathrm{~Pa}$. Lower sublimation pressures result in more faceted morphologies compared with the smooth sublimation fronts achievable close to the equilibrium.

characteristic diffusion and capillary lengths [23]. Specifically, for the solidification of many ice-templated colloidal systems, these characteristic lengths can be ultimately recast such that the general experimental form is also said to follow $\lambda_{o} \propto$ 


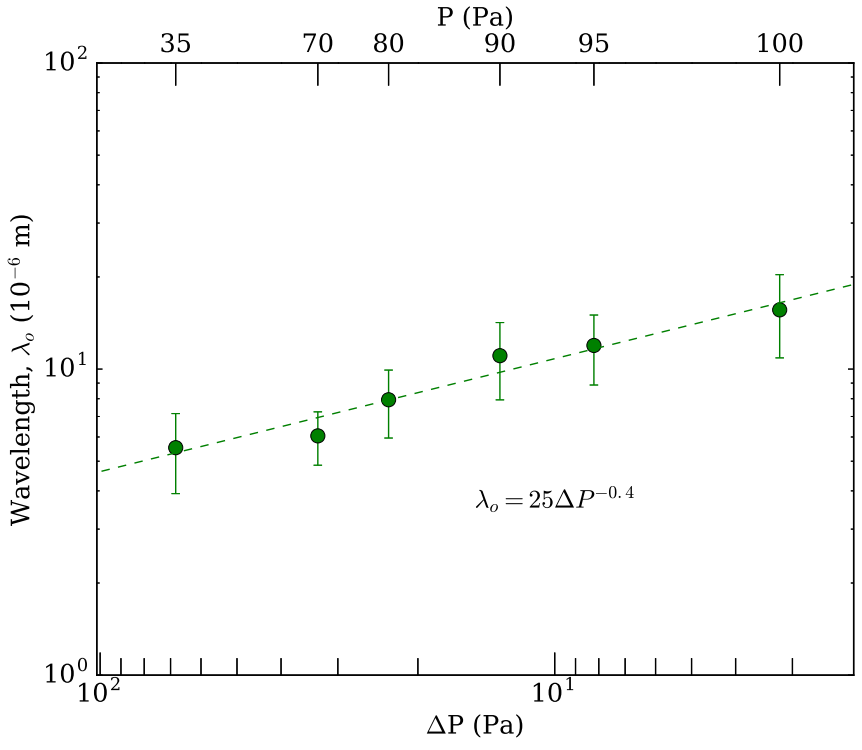

FIG. 5. Logarithmic plot illustrating the relationship between the mean ridge spacings $\lambda_{o}$ and the deviation from equilibrium vapor pressure $\Delta P$ at $-20{ }^{\circ} \mathrm{C} . R^{2}=0.95$, indicating that larger deviations from equilibrium pressures are highly correlated with a refinement of the observed ridge wavelengths, with a suggested exponential relationship between $\Delta P$ and $\lambda_{o}$. The distribution of wavelengths observed is also wider close to the equilibrium, as indicated by the relative sizes of the error bars.
$V^{-n}$ [24,25], similar to the power law observed with the sublimation-induced features.

Discussion. In a similar vein to the Terrace-Ledge-Kink model [26,27], a suggested mechanism for the sublimation of ice is illustrated in Fig. 6. A flat surface has water molecules which initially desorb randomly from the exposed surfaces due to the high density of dangling valences on the surface. As sublimation proceeds, however, more molecules are removed along the basal plane since it is the least enthalpically favored face [8].

Since the basal face has both the lowest density of molecules and the lowest number of violations of the Bernal-Fowler ice rules [28], it requires the least energy to break the hydrogen bonds stabilizing the structure. This results in a basal surface that is atomically rough, but mesoscopically smooth.

However, the removal of molecules from the secondary prismatic plane proceeds via the formation of highly mobile kinks on the adjoining basal planes, owing to the higher stability of the face. The interaction of these kinks and steps compounding on the surface results in the mesoscopic ridges formed along these planes. As the thermodynamic driving force increases, the Lothe-Pound corrected equilibrium defect density [29] arising from such kink formation can be expressed as

$$
\begin{gathered}
n_{\text {defect }} \propto \exp \frac{-\Delta G_{\text {defect }}}{k_{B} T}, \\
\ln \left(p_{e} / p\right) \propto \Delta G_{\text {defect }}=\Delta \sigma+a k T \ln \alpha,
\end{gathered}
$$

\section{Time during sublimation}

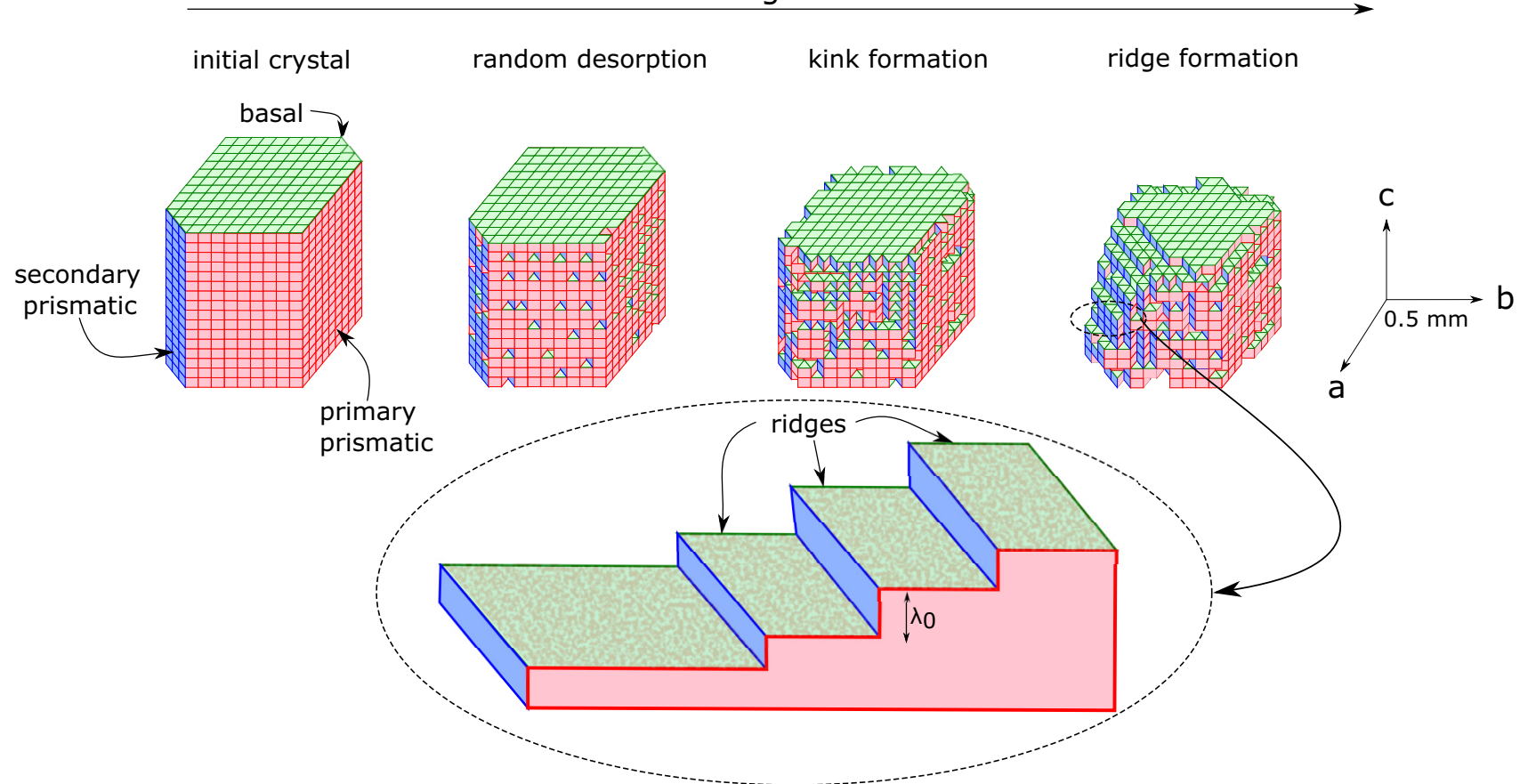

FIG. 6. Suggested mechanism for the preferential crystallographic sublimation on a positive crystal. Initial removal of material is random and is most concentrated along the exposed surfaces and step edges. The basal (green) planes sublime easily resulting in the hollowing of etch pits parallel to the $c$ axis. Sublimation along the secondary prismatic planes is thermodynamically less favorable, resulting in the formation of kinks. The kinks adjoining the basal and prismatic planes form as adatom mobility is impeded by the prismatic plane. These kinks coalesce to form ridges perpendicular to the basal plane and parallel to these secondary prismatic planes (blue). The basal plane remains mesoscopically smooth until the eventual removal of the face, thus resulting in the final sublimation proceeding perpendicular to the secondary prismatic planes. 


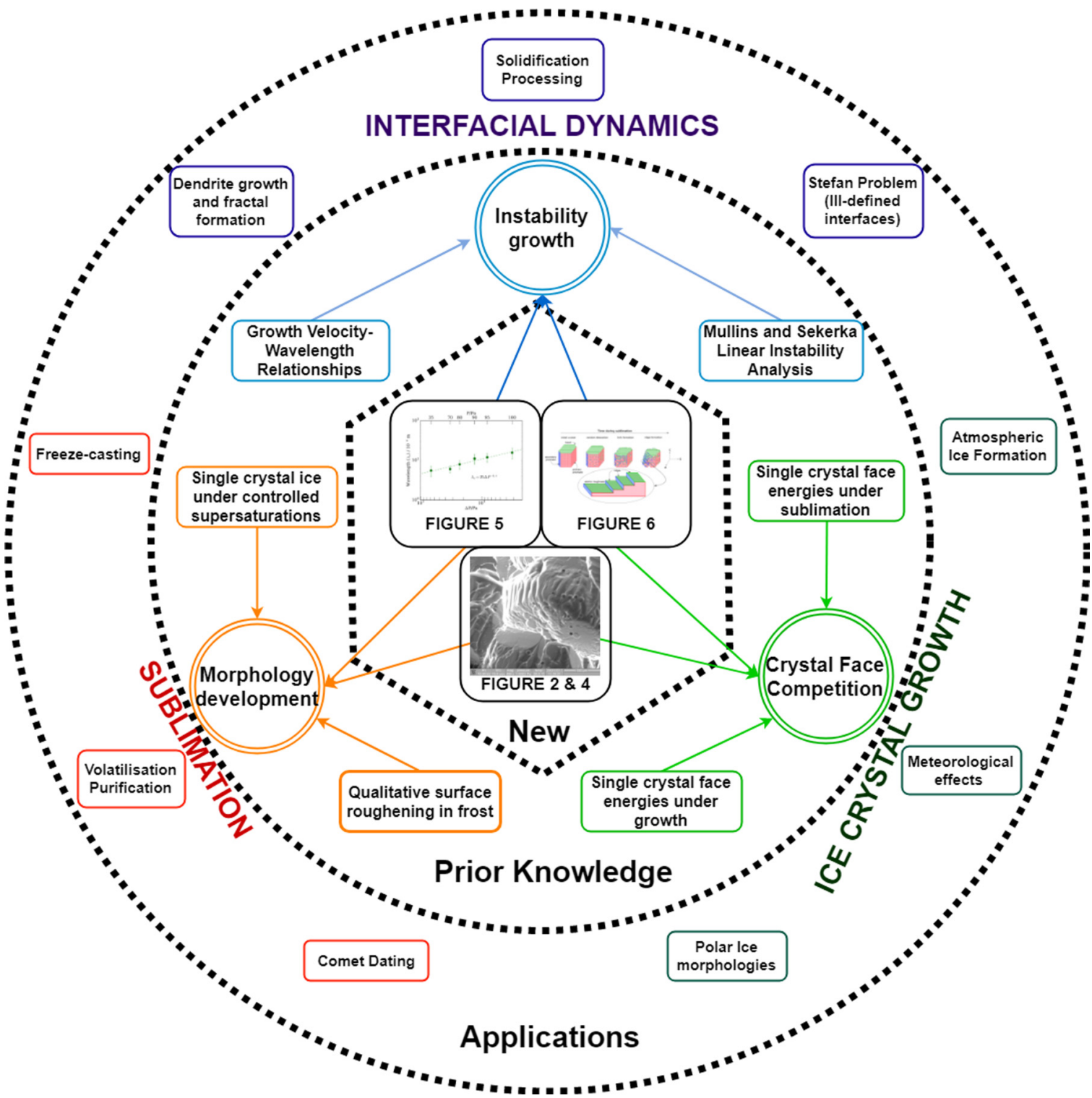

FIG. 7. Contribution to wider disciplines of study. The work done in this paper sheds light on the effect of chamber pressure on the mesostructural faceting observed in ice crystals, and suggests a crystallographic mechanism for this process. These in turn contribute to the pre-existing areas of study in morphological development of crystals, instability growth, and crystal face competition. The combined knowledge can then be used to expand our understanding of ice crystal growth under various conditions, interfacial dynamics through the parametrization of existing theory, as well as the use of sublimation technologies in achieving architectural control or interpreting the origin of structural features.

where $p_{e}$ is the equilibrium vapor pressure, $p$ is the given pressure, $n_{\text {defect }}$ is the defect population density, $\Delta G_{\text {defect }}$ is the energy required to create a defect, $a$ is the number of defect clusters, $\Delta \sigma$ is the change in the surface energy associated with defect formation, $\alpha$ is the attachment or desorption probability, and $k_{B} T$ represents the thermal energy available in the system. As a consequence, although not strictly an absolute underpressure as seen with the results above, the number of ridges that form increases and the wavelength characterizing their equilibrium spacing decreases with a greater drive away from equilibrium.

The presence of these ledges and preference for kinks to form on the basal plane therefore results in the hollowing observed within the crystal morphology as material is removed along intermediary basal planes as opposed to the top surface alone. However, this hollowing along the $c$ axis is not unique to sublimation. Ice growth from vapor has also been suggested to produce hollowed crystals due to the difference in supersaturation along the edges of the crystal in comparison with the center [30].

Upon the removal of these basal planes, only molecules packed along parallel prismatic planes survive, at which point sublimation rates are significantly slowed and the fronts proceed perpendicular to the ridges, reflecting the high energy bonding between the molecules remaining along those planes.

An alternative treatment of the problem may have been sought by considering crystal dynamics approaches rooted either in the theory of step interactions or in interface instability analysis. For the latter, in binary alloy systems and thermally driven dendritic growth, the Mullins-Sekerka linear instability 
analysis is often used to predict the length scale of the observable features [16]. The full details of this analysis is provided in SM Sec. V [12,31-37].

Time series images at $100 \mathrm{~Pa}$ were also obtained (unpublished with this work) with various solutions of acetic acid and sodium chloride to observe the effect of impurities on the morphology and mesostructuring. The images obtained do not exhibit increased mesostructuring with solute concentration, precluding the significance of impurities in the formation of these features in ice.

Conclusions. In summary, this paper has used in situ ESEM imaging to establish the effect of water vapor pressure on the mesoscopic ridges that are observed during sublimation of ice crystals grown from melt. A phenomenological relationship between the underpressure from equilibrium, $\Delta P$, used and the wavelength $\lambda_{o}$ of the periodic ridges that arise in these microstructures was then determined to follow a power law of $\lambda_{o} \propto \Delta P^{-0.4}$. By identifying the crystal etch pits revealed during sublimation, the slow subliming secondary prismatic plane was determined to be the origin of the mesoscopic ridge formation. Subsequently, a crystallographic mechanism was proposed to explain the morphology development through three key steps: initial random desorption, kink formation along the prismatic planes, and finally the subsequent ridge

[1] J. S. Levy, D. R. Marchant, and J. W. Head, Antarctic Sci. 18, 385 (2006).

[2] K. Ivanova and T. P. Ackerman, J. Geophys. Res. Atmos. 114, D06113 (2009).

[3] M. Schieber, R. Carlston, H. Lamonds, P. Randtke, F. Schnepple, and J. Llacer, J. Cryst. Growth 24, 205 (1974).

[4] S. Deville, Adv. Eng. Mater. 10, 155 (2008).

[5] J. Nelson, J. Atmos. Sci. 55, 910 (1998).

[6] W. Beckmann and R. Lacmann, J. Cryst. Growth 58, 433 (1982).

[7] C. A. Knight, J. Appl. Meteorol. 5, 710 (1966).

[8] M. J. Shultz, P. J. Bisson, and A. Brumberg, J. Phys. Chem. B 118, 7972 (2014).

[9] L. Muscariello, F. Rosso, G. Marino, A. Giordano, M. Barbarisi, G. Cafiero, and A. Barbarisi, J. Cell. Physiol. 205, 328 (2005).

[10] G. Danilatos, J. Microsc. 162, 391 (1991).

[11] N. Magee, A. Miller, M. Amaral, and A. Cumiskey, Atmos. Chem. Phys. 14, 12357 (2014).

[12] See Supplemental Material at http://link.aps.org/supplemental/ 10.1103/PhysRevMaterials.2.040401 for additional data (distribution of wavelengths and velocities of front), clarification on terminology used in the paper, and further treatment of data (Mullins-Sekerka).

[13] Y. Takahashi, H. Minoda, Y. Tanishiro, and K. Yagi, Surf. Sci. 433, 512 (1999).

[14] F. Lelarge, Z. Wang, A. Cavanna, F. Laruelle, and B. Etienne, Europhys. Lett. 39, 97 (1997).

[15] D. Kandel and J. D. Weeks, Phys. Rev. B 49, 5554 (1994).

[16] J. S. Langer, Rev. Mod. Phys. 52, 1 (1980).

[17] K. G. Libbrecht, Rep. Prog. Phys. 68, 855 (2005).

[18] J. S. Langer, Phys. Rev. A 36, 3350 (1987).

[19] A. Karma and W.-J. Rappel, Phys. Rev. E 60, 3614 (1999).

[20] G. Demange, H. Zapolsky, R. Patte, and M. Brunel, npj Comput. Mater. 3, 15 (2017). formation due to the coalescence of these kinks. Although we have considered the effect of sublimation on the structure of pure ice, this mesostructuring effect may not be unique to it. As a result, the findings have provided a stepping stone to address other materials that also undergo sublimation or the use of additives within ice itself like collagen [38] or impurities like salts [39]. Thus, as illustrated in Fig. 7, these results thus have the potential to impact a plethora of existing research areas-atmospheric, planetary, and polar sciences as well as sublimation-based processing technologies such as freeze-drying and semiconductor fabrication-where mesostructure is known to affect the observed properties from cloud reflectivity to mesoscale porosity.

Data is available on the University of Cambridge Apollo Open Data Repository at [40].

Acknowledgments. M.N. and A.H. thank J. J. Rickard and E. W. Tapley for their invaluable assistance in using the ESEM. M.N. acknowledges the financial support provided by Gates Cambridge and Geistlich Pharma AG to undertake this research. A.H. and R.E.C. are funded by the ERC Advanced Grant 320598 3D-E. S.M.B. and R.E.C. are funded by the EPSRC Established Career Fellowship Grant No. EP/N019938/1. ESEM imaging was performed at the Cavendish Electron Microscopy suite.

[21] A. Wexler, J. Res. Natl. Bur. Stand. 81A, 5 (1977).

[22] Y. Furukawa and S. Kohata, J. Cryst. Growth 129, 571 (1993).

[23] R. Trivedi and W. Kurz, Acta Metall. Mater. 42, 15 (1994).

[24] S. Deville, E. Saiz, R. K. Nalla, and A. P. Tomsia, Science 311, 515 (2006).

[25] K. M. Pawelec, H. A. van Boxtel, and S. G. Kluijtmans, Mater. Sci. Eng., C 76, 628 (2017).

[26] K. Oura, V. Lifshits, A. Saranin, A. Zotov, and M. Katayama, Surface Science: An Introduction (Springer Science \& Business Media, New York, 2013), Chap. 10.

[27] P. Wynblatt and N. Gjostein, Surf. Sci. 12, 109 (1968).

[28] J. Bernal and R. Fowler, J. Chem. Phys. 1, 515 (1933).

[29] J. Lothe and G. M. Pound, J. Chem. Phys. 36, 2080 (1962).

[30] J. Nelson and M. Baker, J. Geophys. Res. Atmos. 101, 7033 (1996).

[31] H. Zhang, I. Hussain, M. Brust, M. F. Butler, S. P. Rannard, and A. I. Cooper, Nat. Mater. 4, 787 (2005).

[32] W. Macklin and B. Ryan, Philos. Mag. 17, 83 (1968).

[33] C. J. Van Oss, R. F. Giese, R. Wentzek, J. Norris, and E. M. Chuvilin, J. Adhes. Sci. Technol. 6, 503 (1992).

[34] D. James, J. Mater. Sci. 3, 540 (1968).

[35] R. Feistel and W. Wagner, J. Phys. Chem. Ref. Data 35, 1021 (2006).

[36] R. Feistel, paper presented at the IAPWS Meeting, Doorwerth, The Netherlands, September, 2009 (unpublished).

[37] V. Holten, J. V. Sengers, and M. A. Anisimov, J. Phys. Chem. Ref. Data 43, 043101 (2014).

[38] K. M. Pawelec, A. Husmann, S. M. Best, and R. E. Cameron, J. R. Soc. Interface 11, 20130958 (2014).

[39] J. Krausko, J. Runstuk, V. Neděla, P. Klán, and D. Heger, Langmuir 30, 5441 (2014).

[40] https://doi.org/10.17863/CAM.21701. 